\title{
Container deployment strategy for edge networking
}

\section{Wong, W.}

ACM

2019

Wong , W , Zavodovski , A , Zhou , P \& Kangasharju , J 2019 , Container deployment strategy for edge networking . in R Martins , H M C Paulino \& L Veiga (eds), MECC '19:

Proceedings of the 4th Workshop on Middleware for Edge Clouds \& Cloudlets. ACM , pp. 1-6, Workshop on Middleware for Edge Clouds \& Cloudlets , Davis , California , United States , 09/12/2019 . https://doi.org/10.1145/3366614.3368101

http://hdl.handle.net/10138/340347

https://doi.org/10.1145/3366614.3368101

unspecified

acceptedVersion

Downloaded from Helda, University of Helsinki institutional repository.

This is an electronic reprint of the original article.

This reprint may differ from the original in pagination and typographic detail.

Please cite the original version. 


\title{
Bricklayer: Resource Composition on the Spot Market
}

\author{
Walter Wong*, Lorenzo Corneo ${ }^{\dagger}$, Aleksandr Zavodovski*, Pengyuan Zhou*, Nitinder Mohan ${ }^{\S}$, Jussi Kangasharju* \\ ${ }^{*}$ Department of Computer Science, University of Helsinki, Finland \\ Email: \{walter.wong, aleksandr.zavodovski, pengyuan.zhou, jussi.kangasharju\}@ @elsinki.fi \\ ${ }^{\dagger}$ Department of Information Technology, Uppsala University, Sweden \\ Email: lorenzo.corneo@it.uu.se \\ $\S_{\text {Department of Informatics, Technical University Munich, Germany }}$
}

Email: nitinder.mohan@tum.de

\begin{abstract}
AWS offers discounted transient virtual instances as a way to sell unused resources in their data-centers, and users can enjoy up to $90 \%$ discount as compared to the regular on-demand pricing. Despite the economic incentives to purchase these transient instances, they do not come with regular availability SLAs, meaning that they can be evicted at any moment. Hence, the user is responsible for managing the instance availability to meet the application requirements. In this paper, we present Bricklayer, a software tool that assists users to better use transient resources in the cloud, reducing costs for the same amount of resources, and increasing the overall instance availability. Bricklayer searches for possible combinations of smaller and cheaper instances to compose the requested amount of resources while deploying them into different spot markets to reduce the risk of eviction. We implemented and evaluated Bricklayer using 3 months of historical data from AWS and found out that it can reduce up $54 \%$ of the regular spot price and up to $95 \%$ compared to the standard on-demand pricing.
\end{abstract}

Index Terms-Cloud computing, spot instances, availability

\section{INTRODUCTION}

Cloud computing offers many benefits to users, such as flexible on-demand resource allocation and a pay-as-you-go pricing model. One of the main drivers for cloud computing adoption is the reduction of upfront capital investment on infrastructure (CAPEX) by leasing servers in the public cloud as the service grows or scaling servers in the cloud to handle seasonal peak workloads. Cloud providers offer a wide range of virtual server options and subscription models, for instance, reserved, on-demand, and transient instances (known as spot instances in AWS). These spot instances can be up to one order of magnitude cheaper compared to regular prices and, as such, many research works have been done to explore the possible benefits on them in different scenarios, from batch processing [14], [16], [22] to web-serving [11], [13].

Despite the attractiveness of the spot instances, they come with some risks attached, for example, the threat of eviction and the financial risk. Spot instances are sold as instantaneous spare capacity from a cloud provider; thus, they do not enjoy the same Service Level Agreement (SLA) on availability as regular on-demand instances. Cloud providers revoke spot instances to fulfill requests for on-demand servers, which have a higher price point compared to the spot instances. Hence, spot instances can be evicted with a 2-minute notice and, therefore, users need to be aware of this characteristic. The financial risk is due to the nature of the spot market, where prices are driven by supply and demand. Whenever a user bids for a spot instance, the user pays for the current market price of the spot instance rather than the bid price. Therefore, any variations on the price between the market price to the bid price is paid by the user. Our observation of historical spot market data of a 3-month period shows that, on average, the user ends up paying double the lowest market price over the period.

Many research has been done to optimize the use of spot instances, for example, better bidding strategies to get the best pricing and reduce the eviction risk [10], [12], [23], [25], increase application availability by proactively migrating applications between spot and on-demand instances [17], [18], [20] and also strategies to mix-and-match spot and on-demand instances to trade availability for some cost reduction [7]. However, one overlooked issue is the bulk eviction problem, where all spot instances of the same type are evicted simultaneously due to the same bid price. This occurs because many bidding strategies optimize for the best bidding price, and they use multiple instances of the same type and price, thus, whenever the market price goes over the bidding price, all instances are simultaneously evicted from the cloud provider. Another overlooked problem when using spot instances is the application running on top of them, which has different availability requirements and can be broadly divided into two main types: data-intensive applications such as big data and machine learning which can tolerate time delay and some failures [14], [16], [22], [24], and always-on interactive applications, e.g., e-commerce and social networks, which need to be always online.

In this paper, we present Bricklayer, a software tool to assist users in getting the best combination of spot instances that meets the application requirements on cost and availability in the cloud. Rather than receiving a specific spot instance type and finding out the best bidding strategy for it, Bricklayer receives the application resource requirements and constraints and looks for the best spot instance set that can fulfill those requirements, either at optimizing for overall cost or improved availability, allowing for horizontal scaling. Under the hood, Bricklayer checks what is the cheapest AWS EC2 Computing Units (ECU) price for a given virtual hardware family, e.g., CPU or GPU, calculate the price volatility and eviction rates for all instances available in the spot market and return the composed set of spot instances that fulfill the user's requirements. The main benefit of breaking up 
larger resources into smaller ones is that each individual spot instance can be allocated to different spot markets, thus, having different price variations and eviction rates as they now belong to different spot markets. We implemented and evaluated Bricklayer using 3 months of historical spot market data from AWS and evaluated the spot instance composition for the two main categories of application, namely, batch-type and always-on applications. The experimental results show that Bricklayer can achieve up to $54 \%$ discount over the spot instance pricing and up to $95 \%$ discount as compared to regular on-demand pricing on AWS while maintaining the same level of availability.

The paper is divided as follows. Section II presents the background information on AWS spot market. Section III describes the design of Bricklayer. Section IV presents the implementation and the main components of the tool. Section $\mathrm{V}$ shows the evaluation scenarios and the experimental results. Section VI presents the related work and compares them with our work. Finally, Section VII concludes the work.

\section{Spot Instances BACKGROUND}

All major cloud providers offer transient instances as a way to sell under-utilized resources to the customers, for instance, Amazon AWS Spot Instances [3], Google preemptible instances [9], and Azure low priority instances [8]. AWS Spot model uses a dynamic pricing model while Google and Azure use a fixed price model; thus, we will focus our model on the AWS spot market in this paper.

\section{A. Amazon EC2 Spot Market}

AWS offers three subscription models for virtual instances: reserved, on-demand, and spot instances. Reserved instances provide up to $75 \%$ discount compared to the on-demand price [2]. However, the subscriber is bound to a 1 to 3 -year contract (which may or may not be paid upfront). The second option is on-demand, where the user pays for the resource consumption (pay-as-you-go model). In both models, the subscriber has an SLA with AWS where the latter guarantees minimum availability for the virtual machines and also that they are non-revokable, i.e., they cannot be preempted by the cloud provider and have their resources taken back without the subscriber's consent. Lastly, the spot market is where resources are offered with big discounts (up to 90\% off) compared to the on-demand price but without the regular SLA guarantees. Another drawback is the cloud provider can reclaim the resources back after giving just a short notice to the subscriber.

In order to get a spot instance, the customer places a bid in the AWS Spot market. If the bid price is higher than the current market price of the resource, the user will get the instance and pay for the market price. Therefore, the user places a bid with the maximum acceptable price to pay. Once the bid is placed in the spot market, it cannot be changed, and the bid will remain active until the spot instance is evicted or terminated. In case the instance spot market price goes above the bid price, the user will receive a 2 -minute notice

\begin{tabular}{|c|ccc|}
\hline Instance Family & Min. ECU $(\phi / h)$ & Max. ECU $(\phi / h)$ & Diff. (\%) \\
\hline \hline Compute optimized & 0.002600 & 0.008958 & 344.54 \\
\hline General purpose & 0.003154 & 0.011854 & 375.81 \\
\hline Storage optimized & 0.014276 & 0.025212 & 176.60 \\
\hline Memory optimized & 0.003560 & 0.037692 & $1,058.77$ \\
\hline FPGA instances & 0.019038 & 0.021064 & 110.06 \\
\hline GPU instances & 0.022979 & 0.043650 & 189.95 \\
\hline
\end{tabular}

TABLE I: ECU unit price vs. instance family on AWS.

about the virtual machine preemption, and then it will be terminated. There are some works [21], [25] that explore the best bidding strategy at the spot market, leveraging the spot pricing distribution, and choosing the least volatile one. AWS recommends that customers bid for the on-demand price to reduce the chance of eviction [4].

One complexity in the spot market is the number of available options to choose from. The spot market is divided into multiple regions (21 regions), availability zones, instance families and types, resulting in more than $\sim 6 \mathrm{k}$ separate spot markets, where each instance type in a different availability zone has a different spot pricing. Therefore, the same instance in the same region but deployed in a different availability zone may have different prices. In addition to that, spot instances are also divided into several instance families, e.g., generalpurpose (A1, T1) and within each family, their sizings (large, x2.large, etc.).

Despite the attractive pricing for the spot instances, most of the time, they cannot be used out-of-box for most applications. Some instances can be preempted over 10 times a day, affecting application performance and task completion times. Another limitation is that the spot instances can be preempted in groups, i.e., if the market price for an instance type goes over a bid that requested a group of instances, they will be preempted all at once.

\section{B. Spot Market Analysis}

In this section, we will analyze the main metrics that we will use to drive the instance selection decision. For that, we will use 3 months of historical data (March 28th to June 28th, 2019) and analyzed the behavior of three metrics: the EC2 computing unit (ECU) pricing, instance volatility regarding price and the overall instance availability over time.

ECU is a metric provided by AWS to ease the comparison between instance capacity through AWS internal benchmarks. In our case, we will use it to calculate the ECU unit price (1 ECU) for each instance type and pick the cheapest one on the list. Table I compares the ECU unit price for each instance type in different instance families. The results show that ECU unit price has a high variance within the same instance family (up to 10 times) and between different instance families (13 times). Another interesting finding shown in Fig. 1 is that there is no economy of scale on bigger machines (due to higher ECU density) or price increase (as more powerful machines would be more expensive). For that, there is a minimum ECU price that is roughly the same for all machines (except for FPGA and GPU instances). Note that those prices are dynamic 


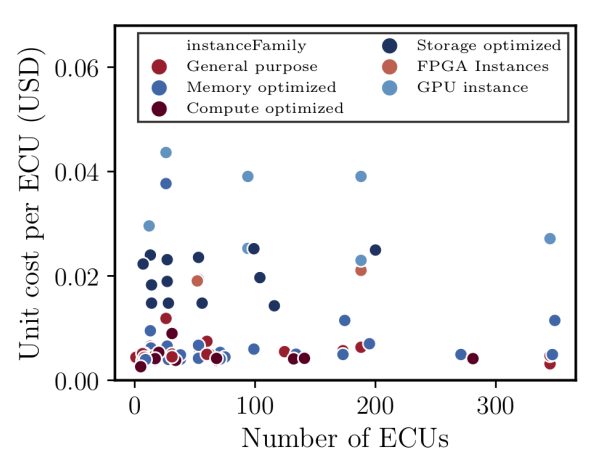

Fig. 1: ECU price vs. instance type

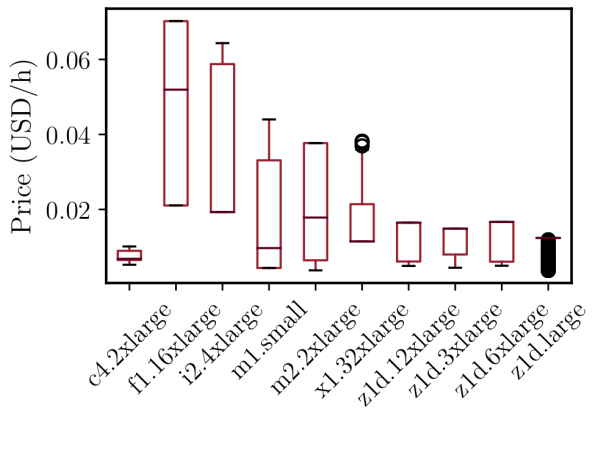

Fig. 2: Volatility (Top 10)

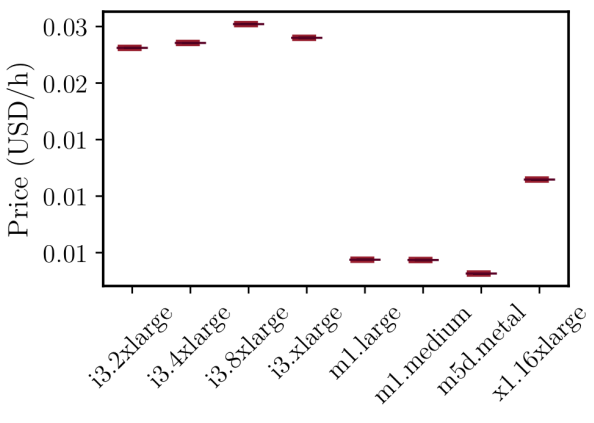

Fig. 3: Volatility (Bottom 10)

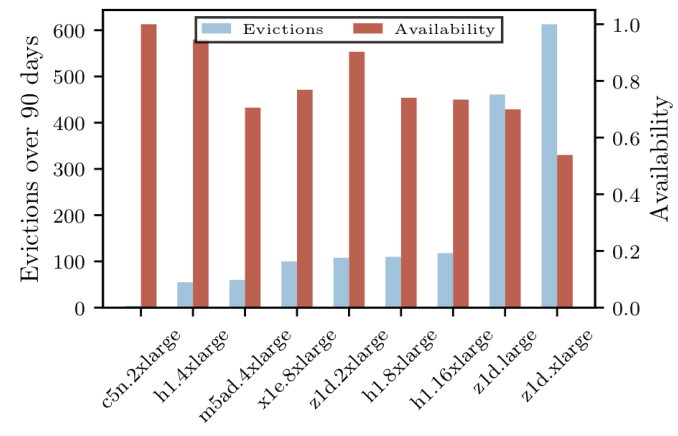

Fig. 4: Number of evictions over 90 days period vs. instance type

and vary throughout the day, and our goal is to pick the cheapest option to minimize the cost on AWS.

Another important conclusion is that there is no difference between getting a bigger machine or a set of smaller machines to compose to a bigger machine. Although the cost is the same, selecting a set of smaller machines from different spot markets to compose to a bigger machine provides higher availability as it reduces the bulk evictions and also the individual eviction rate. Therefore, the main takeaways from the ECU unit price analysis are: (i) select the cheapest ECU unit price instance within the same hardware family, (ii) there is almost no difference between minimum ECU unit price between a small or a large instance on AWS, and (iii) a set of smaller instances are preferred to compose to a bigger instance as they provide higher availability due to different spot markets.

The second metric to be analyzed is the price volatility of each instance. The goal is to select the instances with lower price volatility, so the user doesn't end paying much more than the spot base price. Fig. 2 shows the price volatility of the top 10 instances in the us-east-1 region. Some prices can hike up to $10 x$ (e.g., m2.2xlarge) and also be the same as the on-demand pricing, affecting the overall discounted price that the user may have. Fig. 3 shows instances that did not have changes in the price over the 90 day period, and those are the instances that Bricklayer should preferably select. Therefore, the main takeaway for the second metric analysis is that not all instances are equal in price volatility, and we should focus on those with lower prices and volatility (or higher durability).

The third metric is the instance eviction rate, i.e., the number of times an instance is shutdown by AWS. This metric is important to differentiate the instances with a higher eviction rate (we should avoid them) and the lower eviction rate. Fig. 4 shows the number of evictions for the top 10 most evicted instances. Some instances can be evicted up to 6 times per day, on average. Although some instances may have smaller number of evictions, they can actually present lower availability due to longer periods of downtime. Therefore, the key takeaway is to select instances with lower/lowest eviction rate from the AWS list.

\section{System Design}

The first step of Bricklayer design is to define which kind of applications are going to run on top of it. In one end of the supported application spectrum, we have the time-sensitive, always-on type of application. These always-on applications need minimum downtime as some of them may result in financial losses, e.g., e-commerce and social networks. For example, an outage of 5 minutes costs half-million to Google, and a 10-minute downtime on Amazon may cost 2 million [1], [5]. Therefore, the paramount of always-on applications running over spot instances is to minimize the eviction rate on spot instances.

On the other end of the application spectrum, we have the delay, fault-tolerant, and batch-type applications, where users can tolerate some delays in the job processing time. Some examples in this category include big data processing and machine learning workloads. These kinds of applications can trade some delay for possible reduced costs in the processing; thus, we want to minimize the overall cost of applications running over spot instances.

\section{A. Metrics}

In order to be able to compare and decide which instances are a better fit for each kind of application, we elected the following criteria for spot instance comparison:

ECU unit price. The ECU unit price is the baseline metric to compare the computing power between different instances within the same AWS family type (thus, share the same underlying hardware). It is calculated by dividing the instance's 
hourly price by the number of instance's ECUs. Bricklayer aims to prioritize the selection of instances with the cheapest ECU unit price within the same AWS instance family.

Price volatility. Price volatility represents the amount of variation in the instance price over time, and it is calculated as the relative standard deviation of the instance price. Bricklayer aims to select instances with lower price volatility, which means a lower financial risk of overpaying and more price predictability.

Availability. Availability measures the instance uptime over a given period of time, discounting the number of times the instance has been evicted due to price eviction, i.e., spot instance price going over the on-demand pricing. Bricklayer will choose instances that have lower eviction rates due to price eviction.

With those 3 metrics, Bricklayer can optimize for always-on type of applications (maximize for availability) or batch-type of applications (minimize cost). Next, we will formalize the Bricklayer model, metrics, and the problem statement.

\section{B. Model}

Let be $M=\left\{m: m<N \wedge m \in \mathbb{N}_{+}\right\}$the set of all the available instances provided by AWS, where $N \in \mathbb{N}_{+}$is their availability upper bound. Additionally, we model the set of the $K$ spot markets as $S=\left\{s: s \in \mathbb{N}_{+} \wedge s<K\right\}$. Consequently, we indicate the set of all the available instances in the spot market $s \in S$ with $M_{s}$, that is, $M_{s} \subseteq M$ and therefore $M=$ $\bigcup_{\forall s \in S}\left\{M_{s}\right\}$. We refer to the $i_{t h}$ virtual machine in set $M$ with the notation $m_{i}$. Additionally, $\forall m \in M$ there exists a set of three functions that return, respectively, the instance type, the available number of ECUs and the price per hour. We define instance type function as $t: M \rightarrow \mathbb{N}$ where the return type could be seen as an integer number that maps to a particular instance type defined by AWS, e.g., m2.xlarge. The function $e: M \rightarrow \mathbb{N}$ simply returns the number of ECUs available in a particular instance in $M$. Finally, the hourly price, at time $\tau$, of a spot instance is provided by the function $p:(M, \tau) \rightarrow \mathbb{R}_{+}$. For convenience, we define the set of all the instances of type $i$ as $T_{i}=\{m: m \in M \wedge t(m)=i\}$ and the set of all active instances as $A=\{m: m \in M \wedge o n(m)=1\}$ where on : $M \rightarrow 0,1$ is a binary function returning 1 if the instance is active or 0 otherwise. To be noticed, we use the notation $A_{s}$ to refer to the set of all the active instances in spot market $s$ and, whenever not indicated, it is assumed the set of all active applications regardless of their spot market.

ECU unit price. The ECU unit price, $x$, is a metric defined as the ratio between the hourly price of an instance $m \in M$ and its number of ECU. To put it formally:

$$
x(m, \tau)=\frac{p(m, \tau)}{e(m)}
$$

Price volatility. We calculate the price volatility of $x$ over time by means of its relative standard deviation, that is, the ratio between the standard deviation and the mean. We define the price variability, $v$, of an instance, $m$, as follows:

$$
v(m, \tau)=\frac{\sigma(P)}{\mu(P)}
$$

where $P=\left\{p(m, \tau): \tau \in\left[\tau_{M I N} ; \tau_{M A X}\right]\right\}$, and $\left[\tau_{M I N} ; \tau_{M A X}\right]$ is the time period under investigation subject to price variability calculation.

Availability. The life-cycle of an instance is dictated by the previously defined on function so that we can detect 1-to0 transitions, meaning active-to-evicted, and increment the eviction rate. We introduce the falling edge detection function as $\delta:[\{0,1\}] \rightarrow(0 \mid 1)$, taking as input the values returned by the on function and returns either 0 (evicted) or 1 (active). It is possible to define the eviction rate, $d$, as:

$$
d=\sum_{\forall m \in A} \delta(\text { on }(m))
$$

\section{Problem statement}

We now formalize the problem statement for the always-on and batch-type category of applications. These two types have different objectives and, therefore, two different optimization problems.

Batch-type applications. This category of applications can tolerate some delays and, therefore, the objective is to reduce the ECU unit price of the overall system, $x$, while still satisfying the requirement on the number of ECUs, $E C U_{r e q}$.

$$
\begin{array}{ll}
\underset{\forall m \in A}{\operatorname{minimize}} & x(m, \tau) \\
\text { s.t. } & \sum_{\forall m \in A} e(m)=E C U_{r e q}
\end{array}
$$

Always-on applications. This category of applications have strict uptime requirements and, therefore, the objective is to maximize the overall system availability by selected as many as possible different instances from different spot markets.

$$
\begin{array}{lll}
\underset{\text { s.t. }}{\operatorname{maximize}} & |\hat{S}| & \\
& \hat{S} & \subseteq S \\
& & =\bigcup_{\hat{s} \in \hat{S}} A_{\hat{s}} \\
& \sum_{\forall m \in A} e(m) & =E C U_{r e q}
\end{array}
$$

The result of this maximization is the best set of active instances from different spot markets, $A$, delivering the best possible availability.

\section{IMPLEMENTATION}

We implemented the core Bricklayer features in Python, as shown in Fig. 5. Bricklayer has four main components: (a) Bricklayer engine, responsible for providing the best set of spot instances based on the application type; (b) Bricklayer analytics, responsible for analyzing the spot market pricing; (c) the historical data parser, which fetches AWS spot pricing and stores in the database; and (d) the MongoDB which stores all pricing information and analytics data.

The Bricklayer Engine uses Google Operation Research Tools for the spot instance set combination and optimization based on the application type (always-on vs. batch-type) [6]. The Bricklayer Analytics uses Pandas framework [15] to analyze the historical data and perform the analytics over the metrics. The Historical Data Parser fetches the pricing 


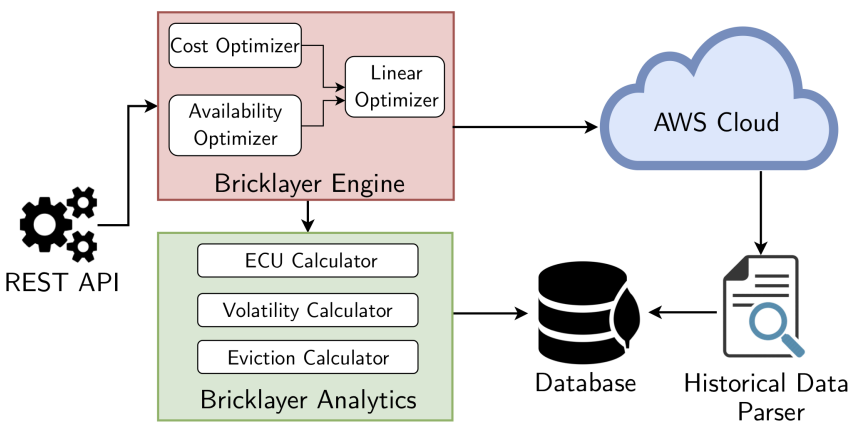

Fig. 5: Bricklayer architectural components.

from AWS and stores it in the MongoDB for the Bricklayer Analytics and the Engine.

Bricklayer interface can be accessed through a REST API in the Bricklayer engine. Users can submit resource requests in JSON format, and the engine will return with the best set of instances for the given application type and also the metrics calculated over the historical data.

\section{Evaluation}

We implemented and evaluated Bricklayer in two types of application scenarios: batch processing and always-on applications. For the input and spot price analysis and optimization, we used the historical spot market data between 29-03-2019 to 29-06-2019 preloaded in the Mongo database. This data is used by Bricklayer Analytics to calculate the ECU, volatility, and eviction rates for each instance in the evaluation period.

We use four popular virtual server instance sizings as a baseline for resource requests to the Bricklayer engine, namely: 16 vcpus (60 ECUs), 32 vcpus (131 ECUs), 64 vcpus (262 ECUs), and 96 vcpus (347 ECUs). For each day, starting on 29-03-2019, we will issue four requests (one for each size) to Bricklayer engine REST API to analyze and provide the best combination of resources to fulfill the requirement.

In order to evaluate the correctness of our implementation, we used Bricklayer to calculate all resource sets resulted from the four requests for all days of the historical dataset. We expect that for all days in the evaluation period, the algorithm will always provide a cheaper price for any request compared to a regular single instance available in the spot market on that given day.

Fig. 6 shows the results of Bricklayer for price optimization compared to regular instance prices for sizes 16, 32, 64 and 96 vcpus. The results show that Bricklayer is able to provide a set of smaller spot instances that can be combined to provide the same virtual resource amount but with 50\% discount, on average, and, in some cases, up to $88 \%$ off compared to regular spot instances. Compared to on-demand instances, Bricklayer can reduce up to $95 \%$ of the regular on-demand price. This is possible because Bricklayer will look for the cheapest instance available at the spot market at a given time and create a resource set on that instance. In some cases, Bricklayer will recommend multiple instances of the same instance type, for instance, 4 instances of the m1.xlarge, if it finds that this is the cheapest price for the ECU over all spot instances, but this may result in lower availability compared to picking up different instance types to compose a resource type. The reason is that the price increase in a given instance type may lead to bulk evictions of all instances. The price optimized resource set is more suited for the fault-tolerant batch type of applications, which can re-run jobs in case of failure without a higher impact on the system. In case the application requires higher availability, Bricklayer allows for trading off cost for higher availability, which we will analyze next.

Fig. 7 shows the spot instance price variation vs. the number of different spot markets. By increasing the number of different spot markets, we reduce the risk of bulk eviction. The reason for that is that the chance of price increase for all virtual instances at a given time is lower compared to a single one, and the change decreases as the number of spot markets increases. For example, in the first figure, having one instance type (e.g., m1.xlarge) will yield the cheapest resource set (in this case, $\$ 0.155 / \mathrm{h}$ ). By having two instance types (or instances in two different spot markets), the price increases to $\$ 0.19 / \mathrm{h}$ for the same amount of processing power. Therefore, users can pay a premium in order to have a resource set with higher availability. On average, choosing more distinct instance types have an increase of $24 \%$ in the first additional distinct instance, and further distinct instances add up roughly $3 \%$. The reason is that Bricklayer is not selecting the cheapest instance type available for the set, but adding the second, third and so forth cheapest instances, resulting in a higher composed price.

Fig. 8 shows the total price of the composed resource set over the 90 day period vs. the number of distinct spot markets (or instances types) and the number of required instances. For this evaluation, Bricklayer only selected the spot instances with the lowest volatility, and eviction rate; thus, all selected instance types in this evaluation have not been evicted during the 3 month period due to price increase. In Fig. 8(a), we compared the total price between regular offthe-shelf standalone spot instances (r4.4xlarge, m5.4xlarge, and r5.4xlarge) with the Bricklayer composite set, shown as opt- $<$ number of distinct instance types $>$. For instance, if the user wants to get the cheapest price option from Bricklayer, she will use 8 instances of the same type (opt- 1 model). However, if she wants to reduce the change of bulk eviction, she can choose instances from different spot markets, which results in higher prices. However, the number of instance may reduce as Bricklayer will get bigger instances to fulfill the requested resource requirements.

\section{RELATED WORK}

HotSpot [18] uses containers within spot instances to migrate the applications between different instances within the same availability zone. It periodically computes the lowest spot price in the market and proactively migrates to a new instance to avoid forced preemption. The main benefit of 

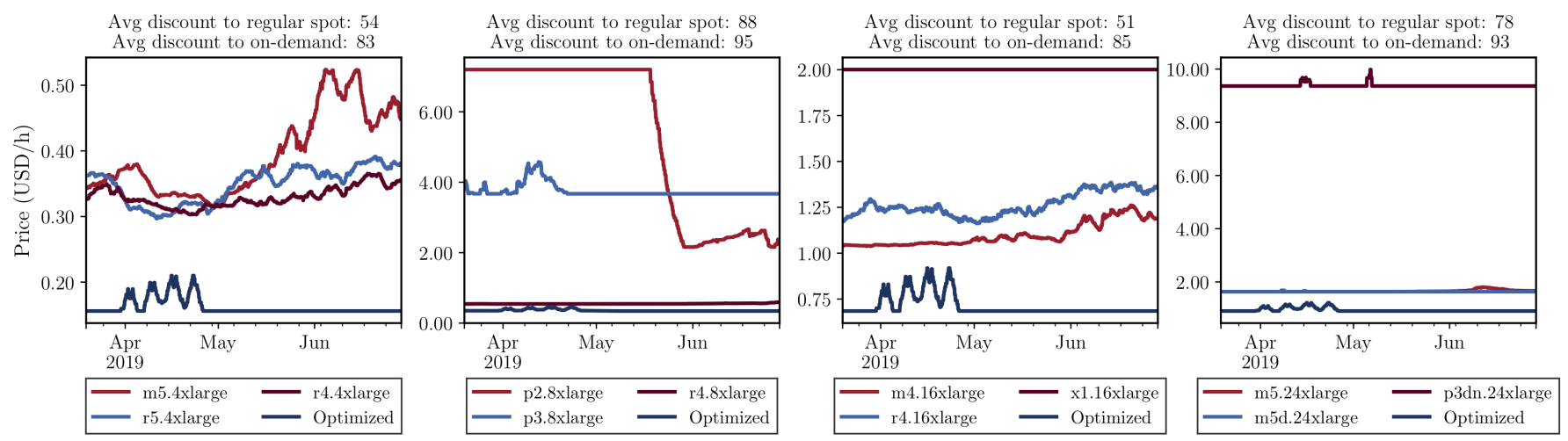

Fig. 6: Cost comparison between the naïve selection of spot instances vs. optimized spot instance selection.
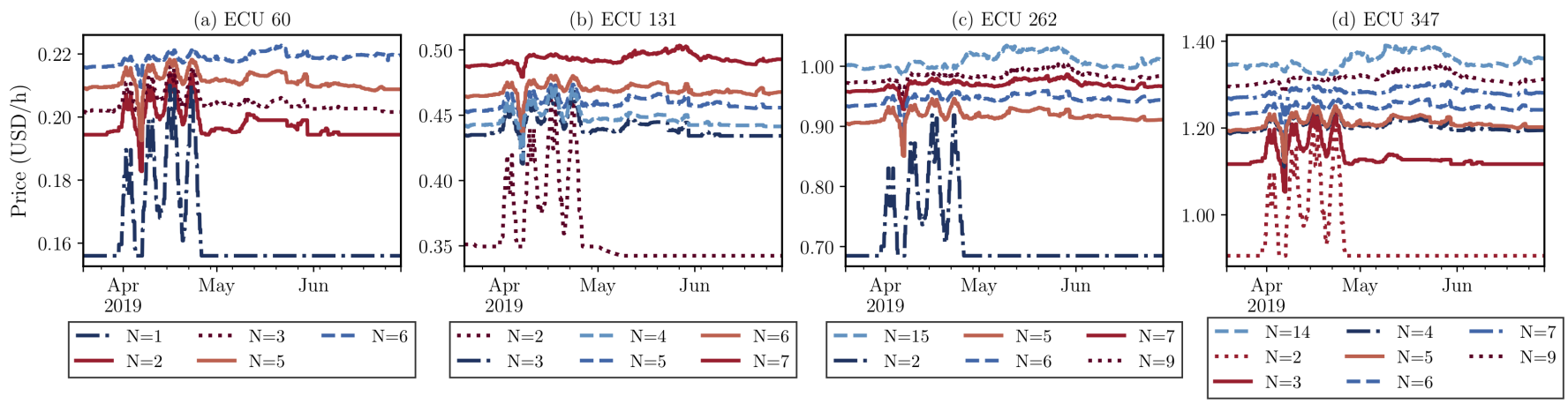

Fig. 7: Cost comparison between number of different spot markets.
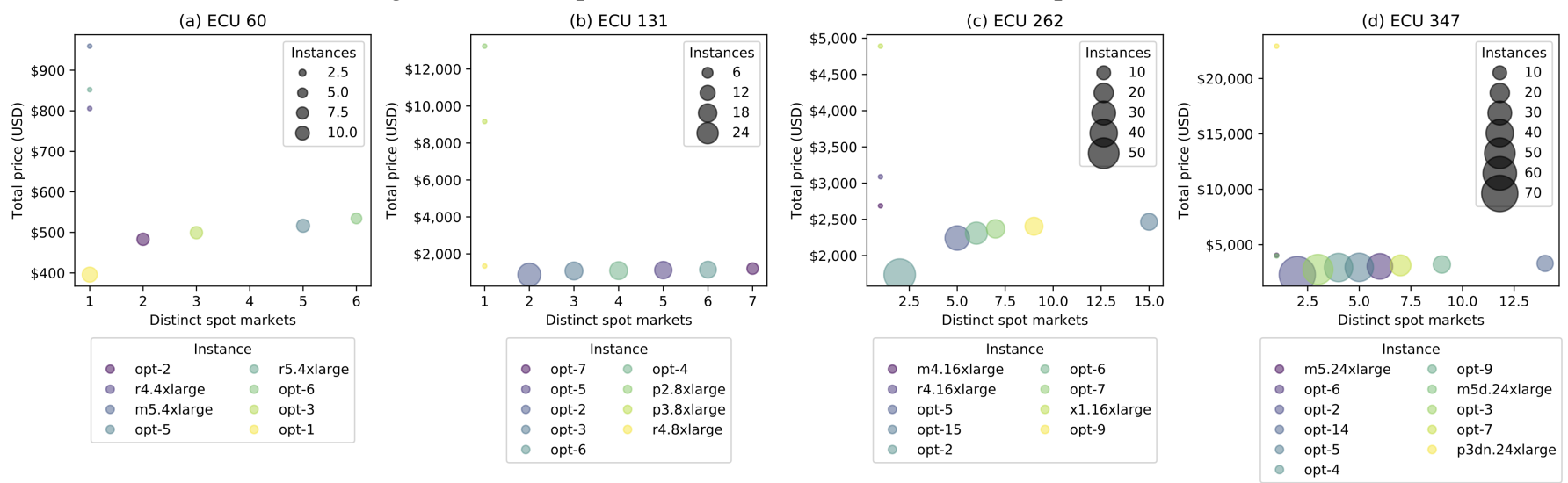

Fig. 8: Comparison between total cost over period of 3 months vs. number of distinct spot markets and number of instances of the same spec. All instances have availability of $100 \%$ as they have not been evicted by price.

HotSpot is it doesn't require any changes to the application inside the container. Compared to Bricklayer, the latter spreads the eviction risk among a set of spot instances, which can be located in different spot markets. With that, the user can reduce even more the risk of eviction compared to the single instance model in HotSpot.

SpotOn [20] is a batch computing service running on top of spot instances, allowing for automatic selection of spot instances and implementing fault tolerance mechanism to mitigate data loss without modifying the application. SpotOn uses containers to encapsulate jobs with their dependencies, and it may use either reactive or proactive container state checkpointing in the disk. In the latter case, the batch computing engine periodically checkpoints the job information in the disk, and, in case of an instance termination, SpotOn can start a container migration to a new instance or deploy a new spot instance and use the last saved checkpoint data. Although Bricklayer does not implement the checkpointing mechanism available on SpotOn, Bricklayer can leverage such mechanism to save intermediate application state during runtime and restoring it in case of failure, resulting in reduced processing time.

SpotCheck [17] describes a derivative cloud market where SpotCheck purchases cloud resources from the big cloud 
providers and resell it with customizations to smaller customers. The contribution is to provide with different solutions and specialized customizations to support customers specific use-cases that are not supported by the original cloud provider. SpotCheck allows running customers' applications within nested VMs inside spot servers whenever possible and transparently migrate to on-demand servers whenever the spot server is revoked. In the similar nested virtualization solution, SuperCloud [19] is a cloud architecture running over OpenStack that integrates multiple cloud providers and allows for live application migration across those providers using Xen virtualization. Supercloud uses nested virtualization to enable the complete VM migration from one server to another, thus allowing users to relocate virtual machines from one cloud provider to another without disrupting the running application.

\section{CONCLUSION}

In this paper, we presented Bricklayer, a resource composition tool over AWS spot instances. Bricklayer gets the resource requirements, constraints, and application type from the user and optimizes for cost or availability. In order to accomplish the optimization process, Bricklayer computes the ECU unit price, price volatility, and overall instance availability for each instance in the spot market and selects the ones that fulfill the requirements. Additionally, Bricklayer can select instances from distinct spot markets to reduce the bulk eviction risk. We implemented and evaluated Bricklayer with AWS historical data, and the results show that the costs can be reduced up to $54 \%$, on average, compared to regular spot instances, and up to $95 \%$ compared to regular on-demand instance prices without compromising the availability aspect.

\section{ACKNOWLEDGMENTS}

This work was supported by the Academy of Finland in the BCDC (314167), AIDA (317086), WMD (313477) projects and by the Swedish Foundation for Strategic Research in the project "Future Factories in the Cloud" (GMT14-0032).

\section{REFERENCES}

[1] 5-minute outage costs Google $\$ 545,000$ in revenue. https://venturebeat. com/2013/08/16/3-minute-outage-costs-google-545000-in-revenue/, 2019. Accessed: 2019-06-01.

[2] Amazon EC2 Reserved Instances Pricing. https://aws.amazon.com/ec2/ pricing/reserved-instances/pricing/, 2019. Accessed: 2019-06-01.

[3] Amazon EC2 Spot Instances. https://aws.amazon.com/ec2/spot/, 2019. Accessed: 2019-06-01.

[4] Amazon Spot Instance Advisor. https://aws.amazon.com/ec2/spot/ instance-advisor/, 2019. Accessed: 2019-06-01.

[5] Cyber Monday: Do You Know the Cost of Your System's Downtime? https://thenewstack.io/ cyber-monday-do-you-know-the-cost-of-your-systems-downtime/, 2019. Accessed: 2019-06-01.

[6] Google Operations Research Tools. https://developers.google.com/ optimization/, 2019. Accessed: 2019-06-01.

[7] How Spot Fleet Works - Amazon Elastic Compute Cloud. https://docs. aws.amazon.com/AWSEC2/latest/UserGuide/spot-fleet.html, 2019. Accessed: 2019-06-01.

[8] Low-priority VMs in Batch. https://azure.microsoft.com/en-us/pricing/ details/batch/, 2019. Accessed: 2019-06-01.

[9] Preemptible VM Instances. https://cloud.google.com/compute/docs/ instances/preemptible, 2019. Accessed: 2019-06-01.
[10] Weichao Guo, Kang Chen, Yongwei Wu, and Weimin Zheng. Bidding for highly available services with low price in spot instance market. In Proceedings of the 24th International Symposium on High-Performance Parallel and Distributed Computing, HPDC '15, pages 191-202, New York, NY, USA, 2015. ACM.

[11] Aaron Harlap, Andrew Chung, Alexey Tumanov, Gregory R. Ganger, and Phillip B. Gibbons. Tributary: spot-dancing for elastic services with latency slos. In 2018 USENIX Annual Technical Conference (USENIX ATC 18), pages 1-14, Boston, MA, July 2018. USENIX Association.

[12] Xin He, Prashant Shenoy, Ramesh Sitaraman, and David Irwin. Cutting the cost of hosting online services using cloud spot markets. In Proceedings of the 24th International Symposium on High-Performance Parallel and Distributed Computing, HPDC '15, pages 207-218, New York, NY, USA, 2015. ACM.

[13] Qin Jia, Zhiming Shen, Weijia Song, Robbert van Renesse, and Hakim Weatherspoon. Smart spot instances for the supercloud. In Proceedings of the 3rd Workshop on CrossCloud Infrastructures \& Platforms, CrossCloud '16, pages 5:1-5:6, New York, NY, USA, 2016. ACM.

[14] Pedro Joaquim, Manuel Bravo, Luís Rodrigues, and Miguel Matos. Hourglass: Leveraging transient resources for time-constrained graph processing in the cloud. In Proceedings of the Fourteenth EuroSys Conference 2019, EuroSys '19, pages 35:1-35:16, New York, NY, USA, 2019. ACM.

[15] Wes McKinney. Data structures for statistical computing in python. In Stéfan van der Walt and Jarrod Millman, editors, Proceedings of the 9th Python in Science Conference, pages 51 - 56, 2010.

[16] Prateek Sharma, Tian Guo, Xin He, David Irwin, and Prashant Shenoy. Flint: Batch-interactive data-intensive processing on transient servers. In Proceedings of the Eleventh European Conference on Computer Systems, EuroSys '16, pages 6:1-6:15, New York, NY, USA, 2016. ACM

[17] Prateek Sharma, Stephen Lee, Tian Guo, David Irwin, and Prashant Shenoy. Spotcheck: Designing a derivative iaas cloud on the spot market. In Proceedings of the Tenth European Conference on Computer Systems, EuroSys '15, pages 16:1-16:15, New York, NY, USA, 2015. ACM.

[18] Supreeth Shastri and David Irwin. Hotspot: Automated server hopping in cloud spot markets. In Proceedings of the 2017 Symposium on Cloud Computing, SoCC '17, pages 493-505, New York, NY, USA, 2017. ACM

[19] Zhiming Shen, Qin Jia, Gur-Eyal Sela, Weijia Song, Hakim Weatherspoon, and Robbert Van Renesse. Supercloud: A library cloud for exploiting cloud diversity. ACM Trans. Comput. Syst., 35(2):6:1-6:33, October 2017.

[20] Supreeth Subramanya, Tian Guo, Prateek Sharma, David Irwin, and Prashant Shenoy. Spoton: A batch computing service for the spot market. In Proceedings of the Sixth ACM Symposium on Cloud Computing, SoCC '15, pages 329-341, New York, NY, USA, 2015. ACM

[21] ShaoJie Tang, Jing Yuan, and Xiang-Yang Li. Towards optimal bidding strategy for amazon ec2 cloud spot instance. In Proceedings of the 2012 IEEE Fifth International Conference on Cloud Computing, CLOUD '12, pages 91-98, Washington, DC, USA, 2012. IEEE Computer Society.

[22] Ying Yan, Yanjie Gao, Yang Chen, Zhongxin Guo, Bole Chen, and Thomas Moscibroda. Tr-spark: Transient computing for big data analytics. In Proceedings of the Seventh ACM Symposium on Cloud Computing, SoCC '16, pages 484-496, New York, NY, USA, 2016. ACM.

[23] Yang Song, M. Zafer, and Kang-Won Lee. Optimal bidding in spot instance market. In 2012 Proceedings IEEE INFOCOM, pages 190198, March 2012.

[24] Chengliang Zhang, Minchen Yu, Wei Wang, and Feng Yan. Mark: Exploiting cloud services for cost-effective, slo-aware machine learning inference serving. In 2019 USENIX Annual Technical Conference (USENIX ATC 19), pages 1049-1062, Renton, WA, July 2019. USENIX Association.

[25] Liang Zheng, Carlee Joe-Wong, Chee Wei Tan, Mung Chiang, and Xinyu Wang. How to bid the cloud. In Proceedings of the 2015 ACM Conference on Special Interest Group on Data Communication, SIGCOMM '15, pages 71-84, New York, NY, USA, 2015. ACM. 